\title{
Presenting mitochondrial antigens: PINK1, Parkin and MDVs steal the show
}

\author{
Cell Research (2016) 26:1180-1181. doi:10.1038/cr.2016.104; published online 2 September 2016
}

In a recent paper published in Cell, Matheoud et al. demonstrated that, in response to cellular stress, selfantigens can be extracted from mitochondria via mitochondrial-derived vesicles and presented at the cell surface to trigger an immune response; this pathway, termed mitochondrial antigen presentation (MitAP), is repressed by PINK1 and Parkin. These findings implicate autoimmune mechanisms in Parkinson's disease.

Parkinson's disease (PD) is a neurodegenerative disorder of unknown etiology. Mutations in PINK1 or Parkin lead to early-onset autosomal recessive $\mathrm{PD}$ and these proteins function together to mediate the lysosomal degradation of damaged mitochondria via autophagy (mitophagy) [1] and of damaged mitochondrial components via mitochondrial-derived vesicles (MDVs) [2, 3]. This implicates a loss of mitochondrial quality control in PD [4]. Matheoud et al. [5] now suggest that a distinct MDV pathway, also regulated by PINK1 and Parkin, may trigger autoimmune responses in PD.

Immune dysregulation, including the presence of activated microglia and upregulation of the expression of inflammatory genes [6, 7], has long been demonstrated in the brains of PD patients. In addition, it has been posited that autoimmune mechanisms may be involved in PD due to high levels of auto-antibodies in patients [8]. However, it is unclear whether these signs of immune or autoimmune activation are a result of the presence of dysregulated neurons or an initiating mechanism of disease.
The work by Matheoud et al. provides a link between PINK1 and Parkin and a potential auto-immune mechanism that could lead to PD. They demonstrate that a novel MDV pathway mediates mitochondrial antigen presentation (MitAP) on major histocompatibility (MHC) class I molecules at the cell surface. MHC class I molecules present intracellular antigens that include viral and tumor peptides, to ensure destruction of cells harboring foreign proteins, and self-antigens. Abnormal responses to self-antigens are implicated in autoimmune diseases. In order to be presented on MHC class I molecules, antigens must be processed to peptides, classically by the proteasome. However, it has recently been shown that antigens can be processed via the lysosome, following autophagy or mitophagy [9, 10]. Matheoud et al. now show that, in addition, mitochondrial proteins can be transported to the lysosome by MDVs for subsequent presentation on MHC class I molecules. Moreover, PINK1 and Parkin negatively regulate this MDV pathway and suppress MitAP. Thus, it is proposed that defects in PINK1 and Parkin might lead to aberrant or overactivation of MitAP, thereby triggering autoimmune mechanisms in PD.

The authors first exploited the antigenicity of glycoprotein B $(\mathrm{gB})$ of herpes simplex virus 1 by targeting it to the mitochondrial matrix (mito-gB). Mito-gB was presented on MHC class I molecules via a mechanism that was dependent on the lysosomal processing of the protein, in contrast to $\mathrm{gB}$ directed to the nuclear envelope (NE-gB) or the cytoplasm (cyto-gB), where only the proteasome was required for antigen presentation. Mito-gB presentation was particularly potently upregulated by heat shock, but was not dependent on autophagy. Furthermore, the response was not mediated by canonical PINK1and Parkin-dependent mitophagy as knockdown of PINK1 potentiated antigen presentation, while Parkin overexpression repressed it. Moreover, treatment with $\mathrm{CCCP}$, a classic trigger of mitophagy, severely dampened the MitAP response.

The autophagy-independent pathway for transporting mitochondrial antigens to the lysosome was demonstrated to be mediated by a novel type of MDV produced in response to heat shock. Previously, PINK1 and Parkin were found to stimulate a distinct MDV pathway, which transports damaged mitochondrial cargo, including the E2/E3 subunits of pyruvate dehydrogenase (PDH) to the lysosome for turnover [2, 3]. In contrast, the MDV pathway described in the current study is repressed by PINK1 and Parkin and appears to function independently of the PDH MDV pathway. Indeed, MitAP is not induced by treatment with antimycin $\mathrm{A}$, which triggers the formation of PDH-positive MDVs, and PDH-positive MDV generation is not stimulated upon heat shock treatment. However, sorting nexin 9 (Snx9) is required for the generation of both types of MDVs, providing a potential link between the two pathways and PINK1 and Parkin (Figure 1). Yet, if PINK1 and Parkin repress MitAP by triggering Snx9 degradation by the proteasome, how do they concurrently stimulate PDH-positive MDV genera- 


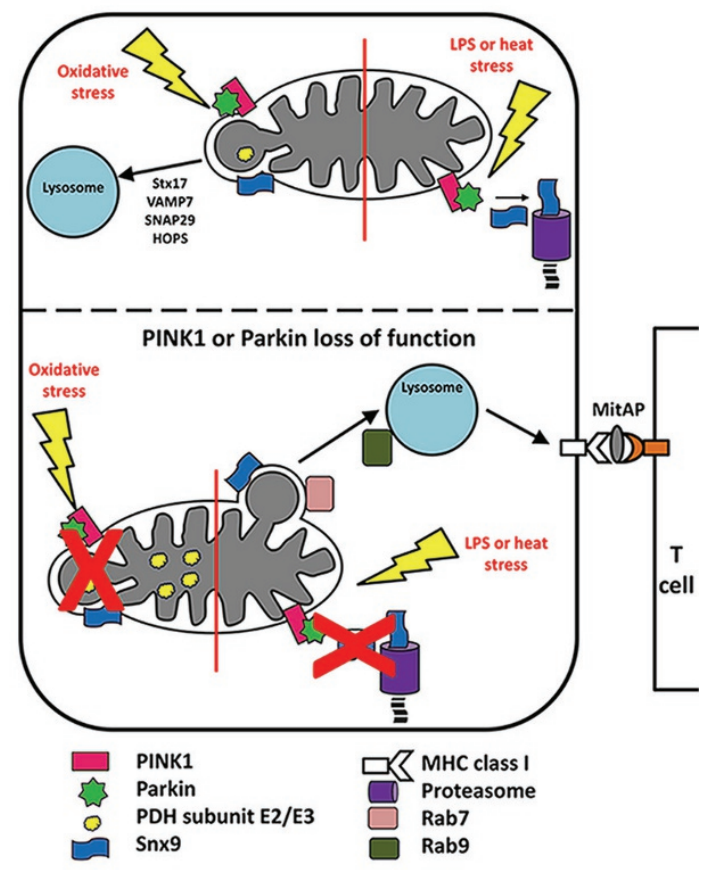

Figure 1 The role of PINK1, Parkin and MDVs in MitAP. (Top panel, right) Under conditions of heat stress or treatment with LPS, PINK1 and Parkin repress MitAP by mediating the proteasomal turnover of Snx9. (Top panel, left) Oxidative stress induces the generation of PDH-positive MDVs containing oxidized and damaged cargo that are directed to lysosomes for degradation. PINK1, Parkin and Snx9 are required for their formation [3, 5] and Stx17, VAMP7, SNAP29 and the HOPS complex are required for their fusion with the lysosome [2]. (Bottom panel, left) In cells lacking functional PINK1 or Parkin, oxidative stress fails to induce the formation of PDH-positive MDVs. (Bottom panel, right) In contrast, treatment with LPS or heat stress in PINK1- or Parkin- null cells induces the formation of MDVs harboring mitochondrial antigens, which requires Snx9 and Rab7 for their formation and Rab9 for fusion with the lysosome. Antigens are processed in the lysosome for presentation on MHC class I molecules at the cell surface. MitAP cognate CD8+ T cells are activated upon binding of their T cell receptors to MHC class I molecules presenting mitochondrial antigens.

tion, which also requires Snx9? It seems likely that there are additional effectors downstream of PINK1 and Parkin that could differentially regulate the biogenesis of these distinct classes of MDVs in response to different stimuli.

Next, Matheoud et al. identified an endogenous mitochondrial protein presented by MitAP, 2-oxoglutarate dehydrogenase (OGDH), a tricarboxylic acid cycle enzyme. The same pathway as dissected for mito-gB is induced to present OGDH peptides on MHC class I molecules, and treatment with LPS in conjunction with IFN $\gamma$ produces similar effects, demonstrating that multiple stimuli associated with infection induce the pathway. Furthermore, the pathway is present in vivo, as mice treated with LPS present OGDH on dendritic cells, an effect enhanced in PINK1- and Parkin-null mice.

The study by Matheoud et al. is an exciting new development in the effort to dissect the complex mechanisms underlying PD. Beyond PD, the results provide novel biological insights into immune regulation and the control of self-antigen presentation, and expands our knowledge of the array of cellular processes utilizing MDVs. In the future, several key biological questions must be addressed, including the identification of additional self-antigens processed by MitAP, particularly in neurons, and elucidation of the signals that allow the lysosome to distinguish MDV cargo to be degraded (i.e., PDH) versus processed for antigen presentation (i.e., OGDH). The exact role of Parkin in the turnover of Snx 9 and whether phospho-ubiquitin, the main product of the PINK1 kinase, is involved in this pathway also need to be determined. Finally, it will be crucial to characterize MitAP in PD patients, starting with isolated dendritic cells from patients with PINK1 or Parkin mutations, as well as in sporadic PD patients. The identification of MitAP and its apparent association with PD may present a paradigm shift in our thinking about the role of immunity and auto-immunity in PD.

\section{Rosalind F Roberts ${ }^{1}$, Edward A Fon ${ }^{1}$}

${ }^{I}$ McGill Parkinson Program, Neurodegenerative Diseases Group and Department of Neurology and Neurosurgery, Montreal Neurological Institute, McGill University, 3801 Rue University, Montréal, Quebec, H3A 2B4, Canada

Correspondence: Edward A Fon

E-mail: ted.fon@mcgill.ca

\section{References}

1 Narendra DP, Jin SM, Tanaka A, et al. PLoS Biol 2010; 8:e1000298.

2 McLelland GL, Lee SA, McBride HM, et al. $J$ Cell Biol 2016; 214:275-291

3 McLelland GL, Soubannier V, Chen CX, et al. EMBO J 2014; 33:282-295.

4 Durcan TM, Fon EA. Genes Dev 2015; 29:989-999.

5 Matheoud D, Sugiura A, Bellemare-Pelletier A, et al. Cell 2016; 166:314-327.

6 McGeer PL, Itagaki S, Boyes BE, et al. Neurology 1988; 38:1285-1291.

7 Mogi M, Harada M, Kondo T, et al. Neurosci Lett 1994; 180:147-150.

8 Barker RA, Cahn AP. Int J Neurosci 1988; 43:1-7.

9 Chemali M, Radtke K, Desjardins M, et al. Cell Mol Life Sci 2011; 68:1533-1541.

10 Bell C, English L, Boulais J, et al. Mol Cell Proteomics 2013; 12:2394-2407. 